\title{
Síndrome de Brugada ou Fenocópia? Desafio Diagnóstico em Paciente com Pectus Excavatum
}

\section{Brugada Syndrome or Phenocopy? Diagnostic Challenge in Patients with} Pectus Excavatum

Kárila Scarduelli Luciano ${ }^{1, *}$, Larissa Freitas Nunes Goldoni ${ }^{1}$, Rafaela Louise Sales ${ }^{1}$, Rafael de March Ronsoni ${ }^{1}$

Luciano KS (D) https://orcid.org/0000-0002-5210-8414

Goldoni LFN (D) https://orcid.org/0000-0003-4340-0349

Sales RL (D) https://orcid.org/0000-0002-5725-279X

Ronsoni RM (D) https://orcid.org/0000-0001-7135-9844

\section{RESUMO}

A Síndrome de Brugada é uma doença cardíaca autossômica dominante causada por disfunções nos canais iônicos, cujos portadores apresentam padrão eletrocardiográfico clássico e predisposição a arritmias ventriculares malignas. As alterações do eletrocardiograma podem ser dinâmicas, espontâneas ou induzidas por drogas bloqueadoras dos canais de sódio (ajmalina, procainamida, etc.). No entanto, os achados eletrocardiográficos que diagnosticam Síndrome de Brugada podem ocorrer mesmo na ausência de disfunção congênita dos canais iônicos, caracterizando "fenocópia de Brugada", portanto a exclusão de causas secundárias é fundamental para o correto diagnóstico da canalopatia.

PALAVRAS-CHAVE: Síndrome de Brugada; Canalopatias; Morte súbita cardíaca.

\begin{abstract}
Brugada syndrome is an autosomal dominant heart disease caused by dysfunctions in the ionic channels, whose carriers present a classic electrocardiographic pattern and predisposition to malignant ventricular arrhythmias. The electrocardiogram alterations may be dynamic, spontaneous, or induced by sodium channel blocking drugs (ajmaline, procainamide, etc.). However, electrocardiographic findings that diagnose Brugada syndrome may occur even in the absence of congenital dysfunction of ionic channels, characterizing "Brugada phenocopy", therefore the exclusion of secondary causes is fundamental for the correct diagnosis of channelopathy.
\end{abstract}

KEYWORDS: Brugada syndrome; Channelopathies; Sudden cardiac death. 


\section{INTRODUÇÃO}

A Síndrome de Brugada (SB) é uma doença autossômica dominante causada por disfunções nos canais iônicos cardíacos. Apresenta padrão eletrocardiográfico diagnóstico chamado tipo 1, determinado por elevação do segmento $\mathrm{ST}>2 \mathrm{~mm}$ em pelo menos uma derivação precordial direita (no segundo, terceiro ou quarto espaço intercostal), seguida por um segmento ST descendente côncavo ou retificado. $\mathrm{O}$ segmento $\mathrm{ST}$ descendente atravessa a linha isoelétrica e é seguido por uma onda $\mathrm{T}$ negativa e simétrica ${ }^{1}$.

O padrão tipo 1 pode ocorrer de modo espontâneo, neste caso geralmente dinâmico, ou induzido por antiarrítmicos bloqueadores do canal de sódio. Embora esse padrão seja fundamental para o diagnóstico, pode ser encontrado na ausência da síndrome genética em algumas condições específicas como pectus excavatum, caracterizando fenocópia de Brugada. Portanto a exclusão de causas secundárias é fundamental para o correto diagnóstico da canalopatia ${ }^{1,2}$.

\section{DESCRIÇÃO DO CASO}

Paciente do sexo masculino, 70 anos, tabagista 50 anos-maço e portador de hipertensão arterial sistêmica com acompanhamento ambulatorial em uso de enalapril $20 \mathrm{mg} / \mathrm{dia}$. Assintomático e sem história pessoal de síncope ou respiração agônica noturna. História familiar de pai com morte súbita aos 42 anos em repouso, sem relato da causa mortis.

Eletrocardiograma de consulta ambulatorial em ritmo sinusal e com V2 de aspecto em sela, compatível com padrão de Brugada tipo 2 (Fig. 1a). Durante exame físico, foi observada a presença de pectus excavatum (Fig. 2b).

Submetido a ecocardiograma transtorácico com átrio esquerdo de aumento moderado, disfunção diastólica de ventrículo esquerdo (VE) grau I com funções sistólicas de VE e ventrículo direito (VD) preservadas e sem evidência de compressão do VD. Espirometria com distúrbio ventilatório obstrutivo moderado sem resposta a broncodilatador, compatível com a história de tabagismo. Tomografia de tórax (Fig. 2a) com índice de Haller normal, estimado em 2,45.

O paciente estava em programação para teste provocativo com bloqueador do canal de sódio, entretanto, em eletrocardiograma seriado em consultas ambulatoriais sequenciais, apresentou padrão dinâmico compatível com tipo 1 (Fig. 1b). Não foi observada fragmentação do QRS.

O padrão dinâmico do eletrocardiograma evoluindo espontaneamente para tipo 1 e a história familiar de morte súbita consolidaram o diagnóstico de SB. Pela idade avançada e ausência de sintomas em fases pregressas sabidamente mais arritmogênicas da síndrome, aliadas à ausência de fragmentação do QRS durante o padrão tipo 1, optou-se apenas em orientações comportamentais e seguimento clínico nesse momento.

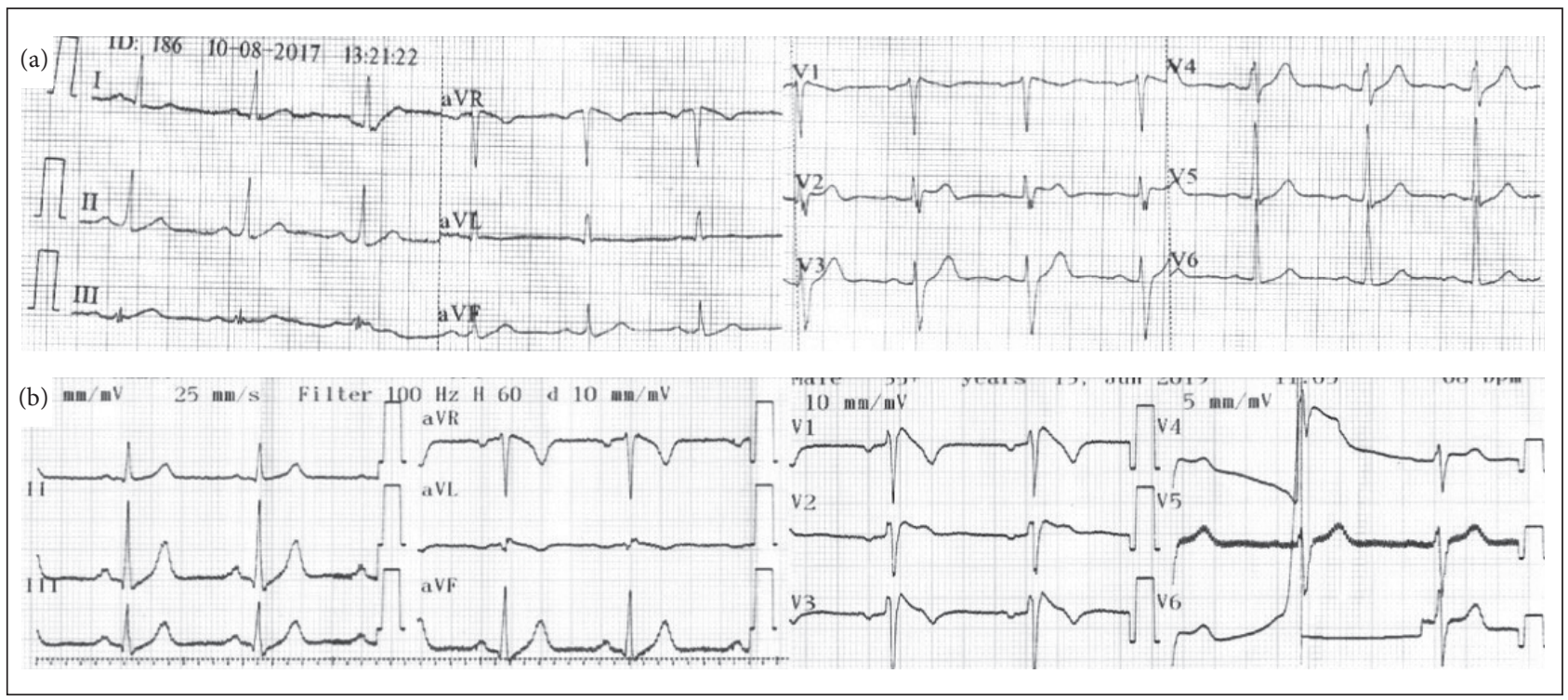

Figura 1. (a) Eletrocardiograma padrão tipo 2 de Brugada e (b) padrão tipo 1 de Brugada. 


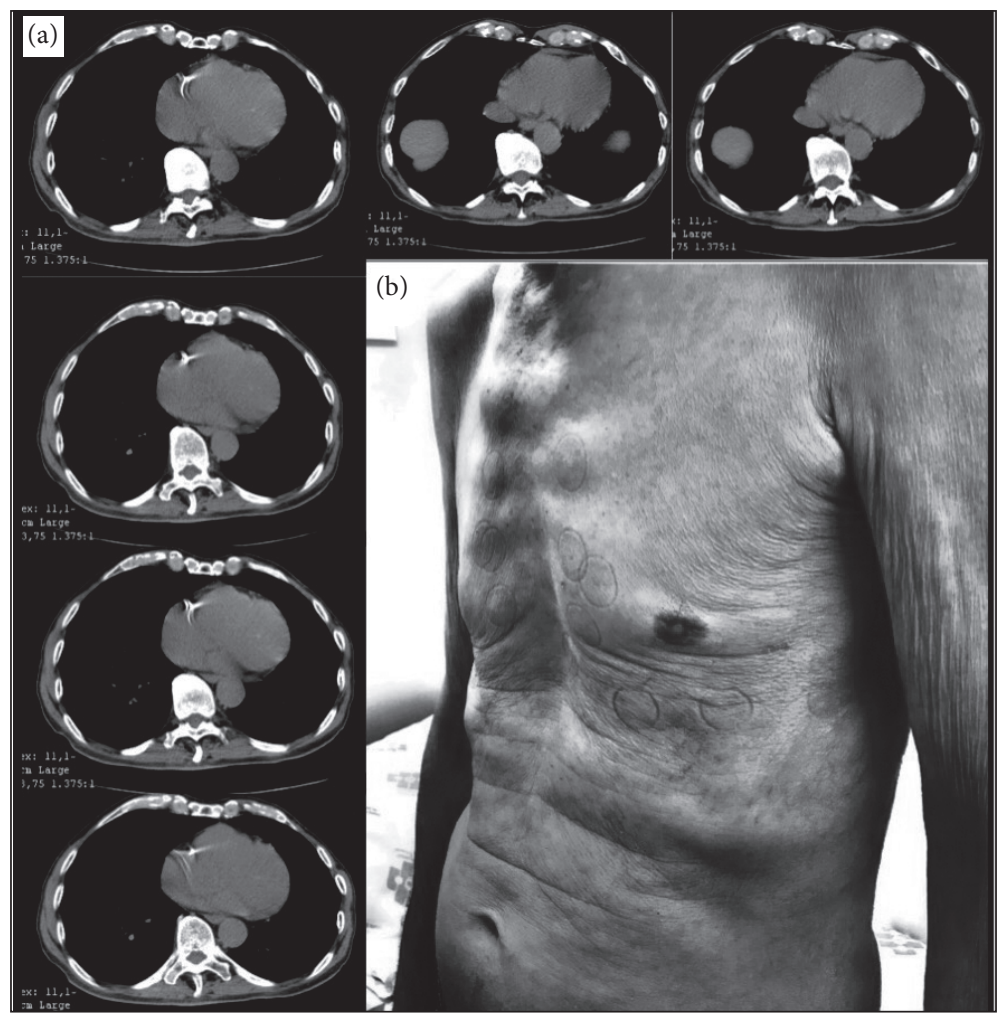

Figura 2. (a) Tomografia computadorizada e (b) exame físico com pectus excavatum.

\section{DISCUSSÃO}

Os achados eletrocardiográficos da SB, tipo 1 ou tipo 2, podem ser encontrados mesmo na ausência de disfunção congênita dos canais iônicos. Esses casos são conhecidos como fenocópia de Brugada e a maioria está relacionada a desequilíbrios metabólicos ou hidroeletrolíticos, isquemia, embolia ou compressão mecânica do $\mathrm{VD}^{1,3}$.

A definição de fenocópia e exclusão da síndrome genética muitas vezes é um desafio em pacientes com pectus excavatum, que podem apresentar padrão tipo 1 ou tipo 2 devido à compressão mecânica do $\mathrm{VD}^{3,5}$.

Não há um marcador eletrocardiográfico para distinguir a fenocópia de Brugada da síndrome genética. Recentemente, foi sugerida uma abordagem em quatro etapas para identificar a possibilidade de se tratar fenocópia em pacientes com o padrão eletrocardiográfico típico de SB na presença de condição clínica que sabidamente poderia influenciar o fenótipo eletrocardiográfico ${ }^{4}$.

Essa abordagem consiste em: 1) identificar o padrão eletrocardiográfico tipo 1 ou 2 da SB;2) determinar se há baixa probabilidade pré-teste de $\mathrm{SB}$, sobretudo relacionada a sintomas do paciente e história familiar; 3) realizar teste farmacológico provocativo com um bloqueador de canal de sódio; 4) realizar testes genéticos (esse critério não é obrigatório) ${ }^{4}$.

Geralmente, os pacientes com fenocópia por pectus excavatum são assintomáticos, não apresentam história familiar de morte súbita e exames complementares evidenciam sinais de compressão extrínseca da parede livre do VD, de forma objetiva em exames de imagem como ecocardiograma avaliando a dinâmica do VD ou de forma subjetiva através de exames complementares que mostram gravidade do pectus excavatum.

A tomografia de tórax é útil em avaliar o grau de depressão esternal e deformação torácica, além do comprometimento cardíaco. Através do índice de Haller, estima-se a gravidade do pectus excavatum, onde valores $>3,25$ denotam compressão severa. Em casos moderados a graves, a espirometria pode evidenciar distúrbio ventilatório restritivo devido à diminuição do volume intratorácico com consequente diminuição dos volumes pulmonares causados pela compressão do pectus excavatum ${ }^{3,5,6}$.

Além disso, pacientes com fenocópia de Brugada por compressão mecânica que permanecem apenas com padrão eletrocardiográfico tipo 2 não evoluem para tipo 1 de forma espontânea ou após uso de antiarrítmicos bloqueadores de canais de sódio ${ }^{1,5}$. 
No caso do nosso paciente, pela idade avançada e por nunca ter apresentado manifestações clínicas da SB, aliado ao exame físico com pectus excavatum, considerou-se como primeira hipótese diagnóstica fenocópia de Brugada. Entretanto os exames complementares não mostraram comprometimento no VD ou mesmo gravidade do pectus excavatum que justificassem a alteração eletrocardiográfica incialmente registrada como padrão tipo 2 de Brugada.

O próximo passo da elucidação diagnóstica seria a realização de teste provocativo com um bloqueador do canal de sódio, para avaliar mudança do padrão tipo 2 para o tipo 1 no diagnóstico da SB. Entretanto, no acompanhamento ambulatorial de forma espontânea e dinâmica, o paciente apresentou padrão tipo 1 , consolidando o diagnóstico da síndrome genética.

Algumas variáveis podem ser implicadas em pior prognóstico e utilizadas na estratificação de risco e prevenção primária de pacientes com SB: padrão tipo 1 espontâneo, história de síncope inexplicada, presença de fragmentação do QRS e estudo eletrofisiológico com período refratário ventricular menor que $200 \mathrm{~ms}^{7}$.

É descrito que pacientes acima de 60 anos com diagnóstico de SB apresentam melhor prognóstico, tanto em relação a eventos primários quanto à ocorrência de choques pelo CDI quando este for indicado para profilaxia secundária por eventos em fases pregressas. Essa melhor evolução parece estar relacionada com a diminuição de testosterona sobre os canais Ito, o que pode contribuir para a baixa incidência de fibrilação ventricular em pacientes idosos com $\mathrm{SB}^{8,9,10}$.

O diagnóstico tardio aos 70 anos de idade, a ausência de sintomas clínicos em fases precoces mais arritmogênicas da SB, a ausência de fragmentação do QRS e o aparecimento efêmero do padrão tipo 1 espontâneo, fez com que nesse momento apenas fossem realizados orientações comportamentais e acompanhamento clínico no nosso paciente. Entretanto, o diagnóstico de SB em idosos não deve ser negligenciado, principalmente devido à importância do rastreamento familiar e impacto clínico na família desses probandos.

\section{CONTRIBUIÇÃO DOS AUTORES}

Conceptualização, Luciano KS; Metodologia, Luciano KS, Ronsoni RM; Redação - Primeira versão, Luciano KS, Ronsoni RM, Goldoni LFN, Sales RL; Redação - Revisão \& Edição, Luciano KS, Ronsoni RM; Aquisição de Financiamento, Goldoni LFN, Sales RL; Recursos, Goldoni LFN, Sales RL; Supervisão, Ronsoni RM.

\section{REFERÊNCIAS}

1. Oliveira-Neto NR, Oliveira WS, Mastrocola F, Sacilotto L. Brugada phenocopy: Mechanisms, diagnosis, and implications. J Eletrocardiol. 2019;55:45-50. https://doi. $\operatorname{org} / 10.1016 /$ j.jelectrocard.2019.04.017

2. Dendramis G. Brugada syndrome and Brugada phenocopy. The importance of a differential diagnosis. Int J Cardiol. 2016;210:25-7. https://doi.org/10.1016/j.jjcard.2016.02.097

3. Awad SF, Barbosa-Barros R, Belem LS, Cavalcante CP, Riera AR, Garcia-Niebla J, et al. Brugada phenocopy in a patient with pectus excavatum: systematic review of the ECG manifestations associated with pectus excavatum. Ann Noninvasive Electrocardiol. 2013;18(5):415-20. https://doi. org/10.1111/anec.12082

4. Gottschalk BH, Anselm DD, Baranchuk A, Xu G. Brugada Phenocopy international registry and online educational portal [Internet]. 2014 [citado 2019 Ago 24]. Disponível em: http://www.brugadaphenocopy.com

5. Marchetti M, Sierecki M, Oriot D, Ghazali A. Brugada-type ECG associated with pectus excavatum. Images Paediatr Cardiol. 2015;17(3):1-2.

6. Abid I, Ewais MM, Marranca J, Jaroszewski DE. Pectus excavatum: A review of diagnosis and current treatment options. J Am Osteopath Assoc. 2017;117(2):106-13. https:// doi.org/10.7556/jaoa.2017.021

7. Priori SG, Gasparini M, Napolitano C, Della BP, Ottonelli AG, Sassone B, et al. Risk stratification in Brugada syndrome: results of the PRELUDE (PRogrammed ELectrical stimUlation preDictive valuE) registry. J Am Coll Cardiol. 2012;59:37-45. https://doi.org/10.1016/j.jacc.2011.08.064

8. Kitamura T, Fukamizu S, Kawamura I, Hojo R, Aoyama Y, Nishizaki $M$, et al. Clinical characteristics and long-term prognosis of senior patients with Brugada syndrome. J Am Coll Cardiol. 2017;3(1):57-67. https://doi.org/10.1016/j.jacep.2016.04.004

9. Conte G, Asmundis C, Sieira J, Levinstein M, Chierchia GB, Giovanni G, et al. Clinical characteristics, management, and prognosis of elderly patients with Brugada syndrome. J Cardiovasc Electrophysiol. 2014;25(5):514-19. https://doi. org/10.1111/jce.12359

10. Kamakura T, Wada M, Nakajima I, Ishibashi K, Miyamoto K, Okamura $\mathrm{H}$, et al. Evaluation of the necessity for cardioverterdefibrillator implantation in elderly patients with Brugada syndrome. Circ Arrhythm Electrophysiol. 2015;8(4):785-91. https://doi.org/10.1161/CIRCEP.114.002705 